\title{
Sixty-five-year-old female with complaint of spontaneous acute pain at the lateral aspect of the knee
}

\author{
Andrea Zagarella $\cdot$ Maria Chiara Castoldi
}

Published online: 5 July 2012

(C) ISS 2012

\begin{abstract}
Answer
Calcific peritendinitis of the lateral gastrocnemius tendon with bony involvement Radiographs show a round, welldefined soft tissue calcification with some heterogeneity within. It is located on the lateral aspect of the external femoral condyle, above the insertion of the popliteal tendon, and associated with subtle cortical erosion of adjacent bone. Eight days later, MRI T2-weighted and STIR images on coronal and axial planes show a cortical-subcortical hypointense lesion surrounded by marked bone marrow edema. The biceps femoris tendon is intact while the gastrocnemius insertion and the more proximal fibers of the collateral lateral ligament show moderate thickening and a mildly increased signal intensity. The surrounding soft tissues are significantly inflamed. Subsequent computed tomography (CT) examination sites the peritendinous calcification between the origin of the lateral gastrocnemius tendon, the lateral collateral ligament, and the biceps femoris.
\end{abstract}

\section{Discussion}

Calcific tendinitis is a self-limiting disease, most frequently found in patients between 40 and 60 years old [1].

The case presentation can be found at http://dx.doi.org.10.1007/ s00256-012-1481-7

\footnotetext{
A. Zagarella $(\bowtie) \cdot$ M. C. Castoldi

Department of Radiology,

Orthopedic Traumatological Center (CTO-ICP),

Via Bignami 1,

20126 Milan, Italy

e-mail: andrea.zagarella@icp.mi.it

M. C. Castoldi

e-mail: maria.castoldi@icp.mi.it
}

Calcifications mostly lie within a tendinous insertion in a tapered fashion. Findings are confined with no mass effect evident. The calcific deposit may also affect peritendinous tissues, making it difficult to recognize its precise origin. Increased signal on MRI T2-weighted images due to inflammatory reaction may be associated. It commonly affects the shoulder joint with predominant location in the supraspinatus tendon. This condition can also occur at other sites involving tendons of the neck, elbow, wrist, hand, hip, and foot [2]. In the knee, the patellar tendon is most often affected while the biceps femoris tendon and popliteus tendon are rarely involved [3-5]. To our knowledge, calcific tendinitis of the lateral gastrocnemius insertion has never been reported.

Calcific tendinitis may lead to erosion of cortical bone, but rarely to cortical breakthrough and bone marrow invasion, which is reported mostly at the shoulder joint [6]. If bone is affected, MRI imaging commonly reveals a focal lesion lodged within bone associated with a marked increase in signal intensity on $\mathrm{T} 2$-weighted images of adjacent bone marrow and soft tissues. At uncommon sites, the lesion may be difficult to distinguish from other pathologies such as infection and neoplasms, and lead to unnecessary biopsies or surgery [6]. When reports demonstrate spontaneous resorption of the calcific lesion within the bone it leaves a cystic cavity [7]; most patients are treated surgically [8-10].

The self-limiting nature of this condition is demonstrated by CT scans obtained at 15 and 60 days after onset, respectively (Fig. 1). The last examination shows complete resorption of the lesion associated with advanced reconstruction of subcortical and cancellous bone.

An eight-month follow-up MRI scan shows normal signal intensity of bone marrow at the affected site and no focal lesion is identifiable within the bone. Surrounding soft tissues are unremarkable (Fig. 2a). Concurrent radiographs show 
Fig. 1 CT scans obtained at 15 (a) and 60 (b) days, respectively after symptom onset
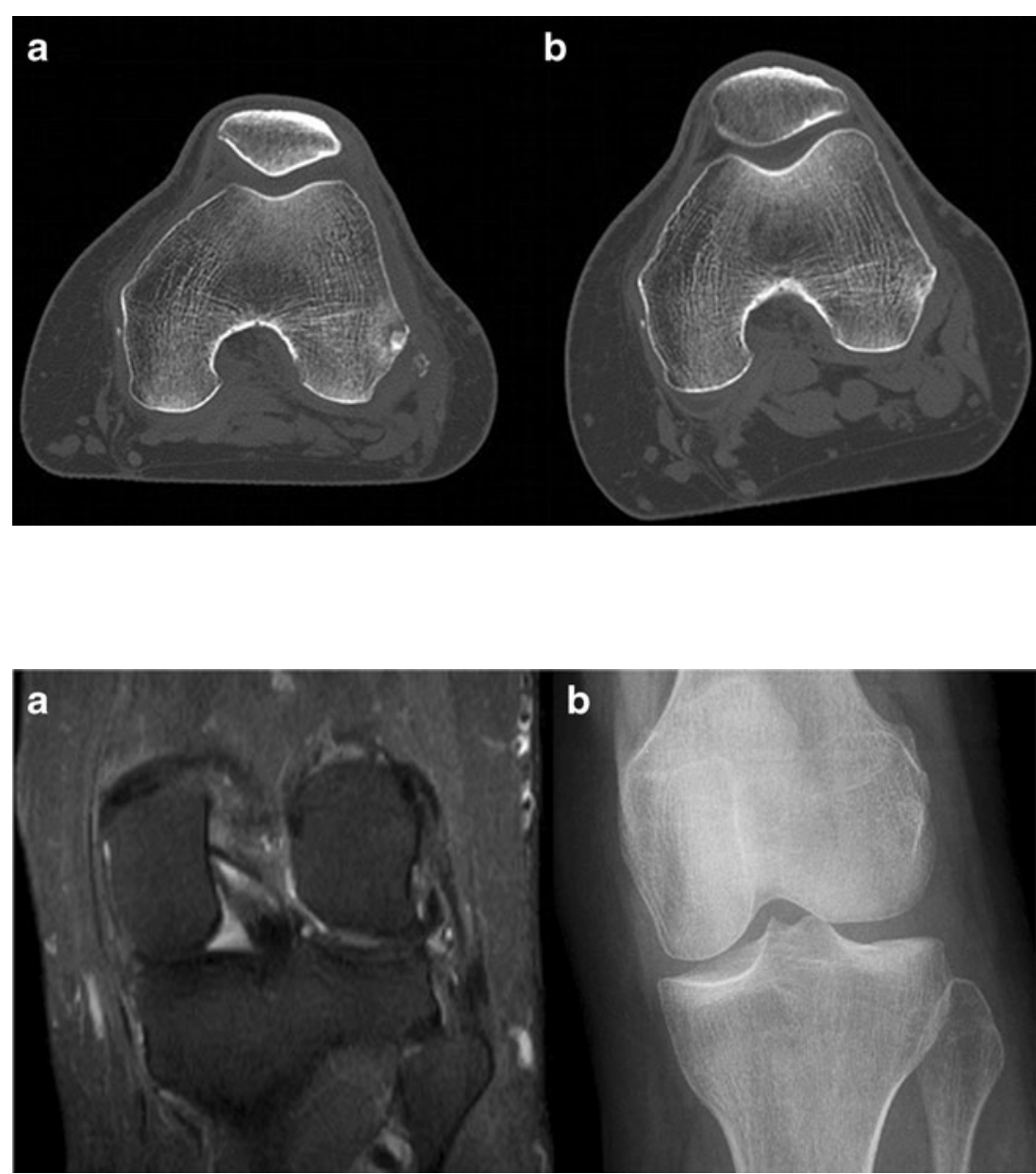

Fig. 2 Eight-month follow-up MRI STIR image (TR/TE/ TI:3600/20/90) coronal plane (a) and radiograph frontal view (b)
3. Tibrewal SB. Acute calcific tendinitis of the popliteus tendon-an unusual site and clinical syndrome. Ann R Coll Surg Engl. 2002;84(5):338-41.

4. Yabe Y, Hatori M, Kumagai J, Koizumi N, Sakuma T, Kawamura M. Heterotopic ossification of the distal portion of biceps femoris: case report and review of the literature. Ups J Med Sci. 2006;111 (3):321-7.

5. Holden NT. Deposition of calcium salts in the popliteus tendon. J Bone Joint Surg Br. 1955;37B(3):446-7.

6. Faure G, Daculsi G. Calcified tendinitis: a review. Ann Rheum Dis. 1983;42(Suppl):49-53.

7. Chagnaud C, Gaubert JY, Champsaur P, Marciano S, Petit P, Moulin G. Vanishing osteosclerotic lesion of the humeral head. Skeletal Radiol. 1998;27(1):50-2.

8. Kraemer EJ, El-Khoury GY. Atypical calcific tendinitis with cortical erosions. Skeletal Radiol. 2000;29(12):690-6.

9. Hayes CW, Rosenthal DI, Plata MJ, Hudson TM. Calcific tendinitis in the unusual sites associated with cortical bone erosion. Am J Roentgenol. 1987;149(5):967-70.

10. Martin S, Rapariz JM. Intraosseous calcium migration in calcifying tendinitis: a rare cause of single sclerotic injury in the humeral head (2010: 2b). Eur Radiol. 2010;20(5):1284-6. 